\title{
Hepadnavirus Genome Replication and Persistence
}

\author{
Jianming $\mathrm{Hu}^{1}$ and Christoph Seeger ${ }^{2}$ \\ ${ }^{1}$ Department of Microbiology and Immunology, Penn State University College of Medicine, Hershey, \\ Pennsylvania 17033 \\ ${ }^{2}$ Fox Chase Cancer Center, Philadelphia, Pennsylvania 19111 \\ Correspondence: juh13@psu.edu; christoph.seeger@fccc.edu
}

\begin{abstract}
Hallmarks of the hepadnavirus replication cycle are the formation of covalently closed circular DNA (cccDNA) and the reverse transcription of a pregenomic RNA (pgRNA) in core particles leading to synthesis of the relaxed circular DNA (rCDNA) genome. cccDNA, the template for viral RNA transcription, is the basis for the persistence of these viruses in infected hepatocytes. In this review, we summarize the current state of knowledge on the mechanisms of hepadnavirus reverse transcription and the biochemical and structural properties of the viral reverse transcriptase (RT). We highlight important gaps in knowledge regarding cccDNA biosynthesis and stability. In addition, we discuss the impact of current antiviral therapies on viral persistence, particularly on cccDNA.
\end{abstract}

$T_{n}^{\text {hen }}$ he animal hepadnaviruses, together with the mammalian foamy viruses and plant caulimoviruses, take a special place within the group of viruses that replicate their genomes with the help of a reverse transcriptase (RT): their genomes are DNA, not RNA. These viruses synthesize DNA in the infected cell "before" the release of infectious, DNA-containing particles, in contrast to conventional retroviruses that perform DNA synthesis immediately "following" infection (Fig. 1). Another feature that sets the hepadnaviruses apart from even its closest relatives lies in the mechanism of RNA packaging and initiation of DNA synthesis, closely linked events that result in a covalent linkage between the first (minus) DNA strand and the RT. The DNA polymerase and RNase $\mathrm{H}$ activi- ties encoded in the RT gene are the only known enzymatic functions specified by hepadnavirus genomes and are major targets for antiviral therapies relying on nucleoside analogs that can suppress, but not cure viral infections. The failure to cure hepatitis B virus (HBV) infections is a consequence of yet another unique property of the hepadnavirus life cycle: the intracellular amplification of the viral covalently closed circular DNA (cccDNA) and its apparent stability in nuclei of infected hepatocytes.

\section{FORMATION OF ccCDNA}

The DNA genome of hepadnaviruses, a relaxed circular DNA (rcDNA), is held together by complementary overlaps that span the region

Editors: Christoph Seeger and Stephen Locarnin

Additional Perspectives on Hepatitis B and Delta Viruses available at www.perspectivesinmedicine.org

Copyright (C) 2015 Cold Spring Harbor Laboratory Press; all rights reserved; doi: 10.1101/cshperspect.a021386

Cite this article as Cold Spring Harb Perspect Med 2015;5:a021386 
J. Hu and C. Seeger



Figure 1. Hepadnavirus life cycle. The figure shows a model for the life cycle of hepadnaviruses RNA- and DNAcontaining capsids are shown in red and blue, respectively. For simplicity, only pregenomic RNA (pgRNA) is shown. ccc, covalently closed circular; dsl, double-stranded linear; env, envelope; rc, relaxed circular. (From Seeger et al. 2013; adapted, with permission, from the authors.)

between the $5^{\prime}$ ends of the two DNA strands (Fig. 2). In mammalian hepadnaviruses, plus strands are shorter than minus strands and have heterogeneous $3^{\prime}$ ends. In avian hepadnaviruses, plus-strand DNA synthesis is almost complete. Following infection of a hepatocyte, the first step in the viral DNA replication cycle is the conversion of rcDNA into cccDNA (Fig. 1). In addition to the extension of plus strands, this process requires removal of the RT from the $5^{\prime}$ end of minus-strand DNA and a primer comprising a capped, $\sim 18$-nucleotide-long RNA derived from the $5^{\prime}$ end of pgRNA (Figs. 2 and 3). In addition, one of the two 9-nucleotide-long terminally redundant segments on minus-strand DNA (r) (Fig. 3D) is removed and the two DNA strands are ligated resulting in the formation of cccDNA.
The exact mechanism for cccDNA formation is still obscure and represents a major gap in our knowledge of the hepadnavirus life cycle. Infection of ducklings with the duck hepatitis $B$ virus (DHBV) revealed that cccDNA formation can occur within a few hours after infection (Tagawa et al. 1986). In theory, cccDNA could be formed by at least two different mechanisms. The first invokes a role for the viral RT. It could be envisioned that the RT, covalently linked to the $5^{\prime}$ end of minus-strand DNA, could facilitate a cleavage-ligation reaction to release itself from the DNA while closing the DNA strand, analogous to the function of the A protein of bacteriophage $\phi \times 174$ during rolling circle DNA replication (Roth et al. 1984). However, mutagenesis-based analyses of the RT, genetic analysis of viral replication, and biochemical studies 


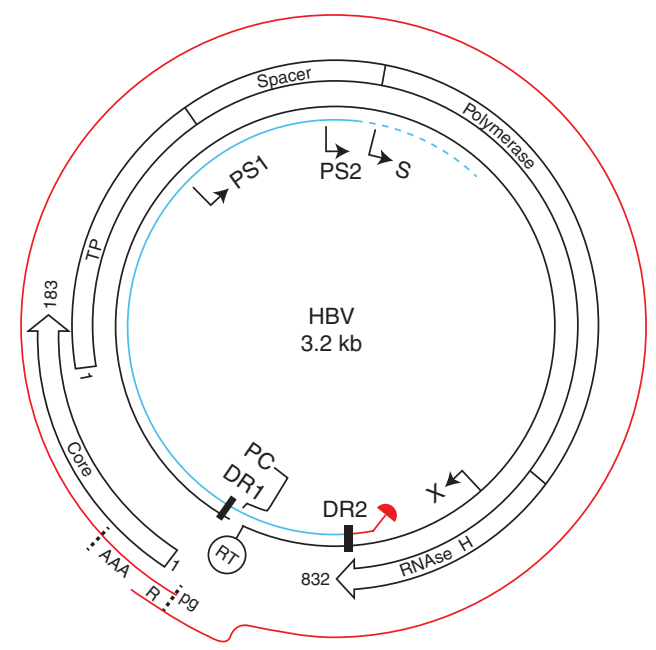

Figure 2. Hepatitis B virus (HBV) genome structure. The relaxed circular DNA (rcDNA) genome of HBV with a complete minus strand (black) and incomplete plus strand (cyan) is shown, together with pregenomic RNA (pgRNA) (red) and the core and pol genes required for DNA replication. Reverse transcriptase (RT) and a capped RNA oligomer at the $5^{\prime}$ ends of minus- and plus-strand DNA, respectively, are indicated. The positions of the start sites for the translation of the precore, presurface $1,-2$, surface, and $\mathrm{X}$ proteins are marked by arrows (PC, PS1, PS2, $S$, and $X$, respectively). $R$, large terminal redundancy on pgRNA; DR, direct repeat; TP, terminal protein of RT.

with RT inhibitors have, so far, failed to provide any evidence for a role of this enzyme in the conversion of rc- to cccDNA (Fourel et al. 1994b; Seeger et al. 1996; Moraleda et al. 1997; Zhu et al. 2001; Sohn et al. 2009). Alternatively, rc- to cccDNA conversion could rely on cellular enzymes normally involved in base excision repair (BER) of DNA damage caused by UV or DNA adducts formed by certain chemicals (Hanawalt and Spivak 2008). Required activities would include an endonuclease, such as XPG, to cleave the viral DNA strands close to their $5^{\prime}$ ends to remove the RT protein and the capped RNA, respectively. A cellular DNA polymerase, such as DNA polymerase $\kappa$, and either ligase I or ligase III could fill in and join the $5^{\prime}$ and $3^{\prime}$ ends of the two DNA strands. Such a model would imply that rcDNA is recognized as "damaged" DNA on delivery into the cell
Hepadnavirus Genome Replication and Persistence

nucleus. Recently, it has been shown that the DNA repair enzyme, Tdp2, a tyrosyl-DNA phosphodiesterase that normally releases covalently trapped topoisomerase II-DNA complex by cleaving precisely at the protein-DNA junction (Cortes Ledesma et al. 2009), can release the RT from the $5^{\prime}$ end of minus-strand DNA in vitro (Jones et al. 2012; Jones and $\mathrm{Hu}$ 2013). It remains to be determined what role, if any, Tdp2 plays in the synthesis of cccDNA in vivo. Interestingly, a protein-free $\mathrm{HBV}$ rcDNA form has been identified in cultured hepatoma cells (Gao and Hu 2007; Guo et al. 2007), but not in the liver or cultured primary hepatocytes (Miller and Robinson 1984; Rumin et al. 1996), suggesting that the mechanisms and pathways used for deproteination and, possibly, cccDNA formation, could vary, depending on the cells used for experiments.

In addition to the canonical pathway for cccDNA formation, a second pathway exists in which double-stranded linear DNA (dslDNA) is a precursor. As will be explained below, dsIDNA is the product of a minor, alternative form of plus-strand priming (Fig. 1). Circularization of dsIDNA occurs by nonhomologous recombination, which leads to the formation of cccDNA with small deletions/insertions, which can nevertheless function as templates for replication of dsl genomes (Yang and Summers 1995, 1998; Yang et al. 1996). dslDNA can also be integrated into chromosomal DNA by illegitimate recombination (Gong et al. 1995). As integrated DNA cannot function as a template for pgRNA, it cannot participate in viral DNA synthesis. However, it can contribute to the development of liver cancer, by activating cellular genes and through the production of truncated surface protein, believed to have oncogenic potential (Seeger et al. 2013).

Once formed, cccDNA is transcribed from four promoters into $\mathrm{pg}$, presurface (preS), surface (S), and X RNAs (Figs. 1 and 2). pgRNA is the template for the synthesis of the two proteins required for viral DNA synthesis, core, and RT. Following translation, pgRNA serves as the template for reverse transcription of minus-strand DNA, as will be described in detail in the following sections. 
J. Hu and C. Seeger

\section{RNA PACKAGING AND ASSEMBLY OF THE REPLICATION COMPLEX}

A critical, but still not-well-understood step in the replication cycle of hepadnaviruses is the sequestration of pgRNA from the translation machinery, which coincides with or is followed by pgRNA packaging into core particles (Fig. 1). Packaging of pgRNA depends on the binding of the RT to a stem-loop structure, termed epsilon ( $\varepsilon$ ) near the $5^{\prime}$ end of pgRNA (Figs. 3 and 4 ) (Bartenschlager et al. 1990; Hirsch et al. 1990; Junker-Niepmann et al. 1990). A second copy of $\varepsilon$ present at the $3^{\prime}$ end of the RNA does not play any role in packaging or DNA replication. In avihepadnaviruses, packaging requires a second RNA segment that overlaps the beginning of the pol gene (Calvert and Summers 1994). How it contributes to the assembly process is not known. Reverse genetic experiments revealed that pgRNAs that encode a functional pol gene are preferentially encapsidated, suggesting that translation and sequestration of pgRNA are cou- pled events (Bartenschlager et al. 1990). Consistent with such a model are results indicating that the cap structure at the $5^{\prime}$ end of pgRNA is required for packaging, suggesting that the RT could interact with the translation machinery to induce the packaging process (Jeong et al. 2000).

Cellular proteins, belonging to the family of molecular chaperones, are required for the initial binding of the polymerase to $\varepsilon$ RNA (discussed below). This interaction, then, activates the protein-priming reaction for minus-strand DNA synthesis and the assembly of core proteins into capsids. The exact sequence of events of the RNA-packaging and protein-priming reactions is not well understood because, to date, only the latter reaction could be reconstituted in vitro. The protein-priming reaction comprises formation of a covalent linkage between a tyrosine residue, located in the amino-terminal domain of the RT, termed terminal protein (TP), and dGMP (deoxyguanosine monophosphate) (Weber et al. 1994; Zoulim and Seeger 1994). As

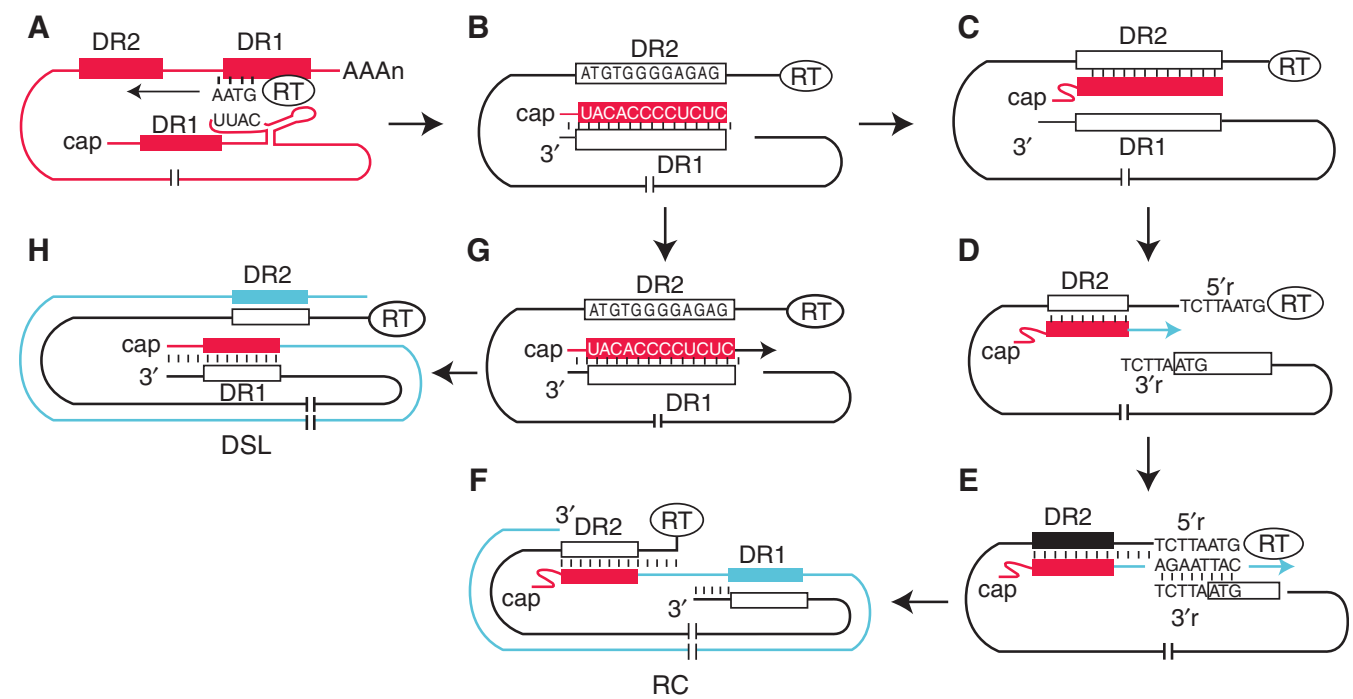

Figure 3. DNA replication cycle. The figure shows a model for the formation of relaxed circular DNA (rcDNA) and double-stranded linear (dsl) from pregenomic RNA (pgRNA). (A) Transfer of the DNA primer from $\varepsilon$ to DR1 near the $3^{\prime}$ end of pgRNA, $(B)$ synthesis of minus-strand DNA and digestion of pgRNA by RNase $\mathrm{H},(C)$ transfer of the capped RNA primer from DR1 to DR2, $(D)$ synthesis of plus-strand DNA to the $5^{\prime}$ end of minusstrand DNA, $(E)$ template switch of the nascent plus strand with the help of the small terminal redundancies, $5^{\prime} \mathrm{r}$ and $3^{\prime} \mathrm{r}$, resulting in circularization of the genome, $(F)$ completion of plus-strand DNA synthesis, $(G)$ in situ priming of plus-strand DNA, and $(H)$ formation of dsIDNA. 


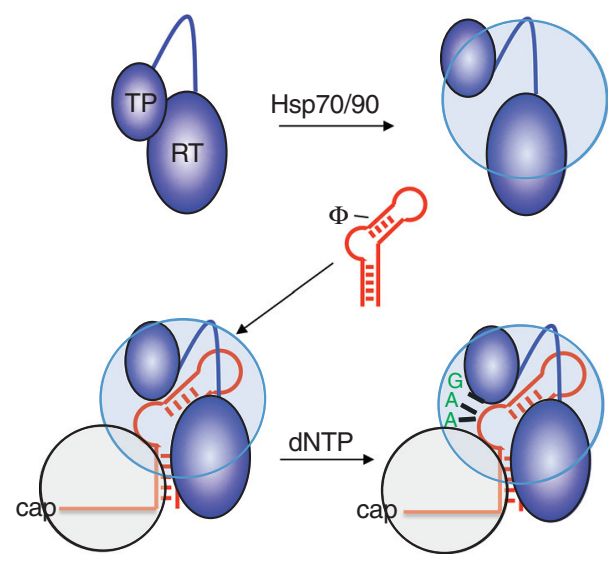

Figure 4. Ribonucleoprotein complex for the proteinpriming reaction. The figure shows a model for the first steps of pregenomic RNA (pgRNA) packaging and protein priming of reverse transcription. Binding of $\varepsilon$ RNA to the terminal protein (TP) and polymerase domains of the reverse transcriptase (RT) is facilitated by chaperones (blue circle). $\Phi$ depicts the RNA segment that is believed to base pair with $\varepsilon$ and is required for circularization of pgRNA. Additional protein factors believed to play a role in RNA packaging by binding to the cap structure are indicated (gray circle). dNTP, deoxyribonucleotide triphosphate; Hsp90, heat shock protein 90.

a consequence of this covalent attachment, hepadnaviral polymerases, in contrast to retroviral RTs, are template specific. Moreover, the nucleocapsid assembly mechanism of hepadnaviruses favors packaging of pgRNAs that encode structurally and functionally intact polymerase, further contributing to accurate propagation of the viral genome. To complete the priming reaction, the RT copies 3-4 nucleotides from the bulge of $\varepsilon$ RNA (Figs. 3 and 4 ) and remains covalently linked to the $5^{\prime}$ end of minus-strand DNA during all subsequent steps of viral DNA synthesis (Gerlich and Robinson 1980; MolnarKimber et al. 1983). DHBV capsids in duck livers contain capsids with minus strands of at least 30 nucleotides in length, supporting the view that the very early stage of viral DNA synthesis takes place inside core particles (Molnar-Kimber et al. 1983). On the other hand, experiments with DHBV capsids isolated from tissue culture cells treated with an RT inhibitor suggest that pgRNA and RT first assemble with
Hepadnavirus Genome Replication and Persistence

core subunits to form a metastable complex for the DNA-priming reaction, which is then converted to a stable capsid during the elongation of minus strands (Guo et al. 2003). Interestingly, packaging of pgRNA is, at least, partially inhibited in cells treated with interferon (IFN), further suggesting that several unknown parameters control this complex mechanism (Guo et al. 2003).

\section{VIRAL DNA SYNTHESIS}

To continue DNA synthesis, the 3-4-nucleotide-long DNA oligomer linked to the RT is transferred to the $3^{\prime}$ end of pgRNA, where it anneals with a complementary sequence motif located within a 10-12-nucleotide-long region known as DR1 (Fig. 3) (Wang and Seeger 1993). As expected, the 3- to 4-nucleotide acceptor site by itself is insufficient to specify the transfer to DR1. This step requires additional sequences on pgRNA. Moreover, acceptor and donor sites are likely held in close physical proximity to facilitate the transfer of the oligomer across the pregenome. Consistent with such a model, a short cis-acting element, termed $\phi$, was identified upstream of the acceptor site at DR1 (Figs. 3 and 4) (Abraham and Loeb 2006; Maguire and Loeb 2010). Sequences in $\phi$ can base pair with the $5^{\prime}$ region of $\varepsilon$, thereby stabilizing a circular conformation of pgRNA and facilitating the transfer of the short minus-strand DNA. In addition, $\phi$ RNA can anneal with another RNA sequence, termed $\omega$, which is located downstream from DR1, and is also required for minus-strand DNA synthesis (Abraham and Loeb 2007).

Following the translocation reaction, minus-strand DNA synthesis continues all the way to the $5^{\prime}$ end of pgRNA (Fig. 2), which is concomitantly degraded by the RNase $\mathrm{H}$ activity of the RT (Summers and Mason 1982). Because of the location of DR1 within the large terminal redundancy on pgRNA, minus strands bear short 9-nucleotide-long terminal redundancies, termed $r$, that play an important role in the circularization of the viral genome, which facilitates plus-strand DNA synthesis (Seeger et al. 1986; Lien et al. 1987). 
Plus-strand DNA synthesis is primed by an 18-nucleotide-long, capped RNA oligomer derived from the $5^{\prime}$ end of pgRNA following its degradation by RNase $\mathrm{H}$. The $3^{\prime}$ portion of this RNA coincides with DR1, which is essential for annealing to complementary sequences at DR2 near the $5^{\prime}$ end of the minus-strand DNA (Fig. 3) (Lien et al. 1986; Seeger et al. 1986; Loeb et al. 1991). As expected, mutations that disrupt the homology between DR1 and DR2 block the formation of rcDNA and, instead, favor an in situ DNA-priming reaction that leads to the formation of dsIDNA (Figs. 1 and 3) (Staprans et al. 1991). The in situ priming reaction also occurs under normal conditions with a frequency of $\sim 5 \%-20 \%$ relative to the translocation event described for rcDNA formation. In addition to the direct repeats, structural features of minus strands in core particles most likely promote the transfer of the RNA primer across the genome. Studies with DHBV and HBV revealed the presence of three sequence motifs in minus strands that could form short duplexes, which stabilize a secondary structure required for the translocation of the RNA (Liu et al. 2003; Lewellyn and Loeb 2007). Mutations that disrupt the formation of these duplexes inhibited rcDNA, but not dsIDNA synthesis (Liu et al. 2003).

Following the priming reaction at DR2, plus-strand DNA synthesis progresses to the $5^{\prime}$ end of the minus-strand DNA, where a template switch is again required for the continuation of DNA synthesis (Fig. 3). This switch is responsible for circularization of the genome and depends on the aforementioned terminally redundant sequences, $r$, in the minus-strand DNA. As with priming at DR2, this step also depends on the formation of small duplexes at distant sites in the minus-strand DNA, indicating that these two critical reactions in plus-strand DNA synthesis might be controlled by similar mechanisms (Mueller-Hill and Loeb 2002).

In mammalian hepadnaviruses, plus-strand DNA synthesis in core particles arrests to varying degrees when approximately half of the genome has been copied from minus strands, producing plus strands with heterogeneous $3^{\prime}$ ends (Summers et al. 1975). This arrest in DNA synthesis could be caused by constraints imposed by the capsid and/or the RT. The fact that plus-strand DNA synthesis in DHBV is complete under natural conditions suggests that the HBV capsid structure may be responsible for arresting plusstrand DNA synthesis (Lien et al. 1987). The influence of capsid structure on plus-strand DNA synthesis has also been shown with genetic experiments. For example, DHBV capsids assembled from core proteins with truncated carboxy termini, or substitutions that mimic constitutive phosphorylation of certain serine residues, also show defects in plus-strand DNA elongation (Yu and Summers 1991; Basagoudanavar et al. 2007). Alternatively or additionally, it is conceivable that the nucleotide triphosphate pool in HBV capsids is depleted before completion of the plus-strand DNA. This possibility is supported by the demonstration that plus-strand DNA can be extended in an in vitro reaction in the presence of nucleotide substrates and nonionic detergents that disrupt the viral envelope (Kaplan et al. 1973; Summers et al. 1975).

As a consequence of DNA synthesis, capsids acquire the signals necessary for assembly with envelope proteins and the subsequent egress from infected hepatocytes (Summers and Mason 1982; Gerelsaikhan et al. 1996; Wei et al. 1996; Perlman and $\mathrm{Hu}$ 2003). Paradoxically, HBV capsids containing no RNA or DNA are assembled and secreted from hepatocytes and hepatoma cells as genome-free, empty enveloped particles that are in large excess over complete, DNA-containing virions (Kaplan et al. 1976; Ning et al. 2011). DNA-containing capsids can also enter a retrograde pathway that delivers rcDNA into the cell nucleus (Tuttleman et al. 1986; Wu et al. 1990). In DHBV, envelope proteins play a major role in regulating the fate of capsids (Summers et al. 1990, 1991; Lenhoff and Summers 1994). Early in infection, when envelope protein levels are low, the retrograde transport pathway is favored over the virion assembly pathway, leading to the accumulation of 5-30 copies of cccDNA per infected hepatocyte nucleus (Fig. 1). It should be noted that cccDNA cannot replicate in a semiconservative manner and, hence, reverse transcription is the only known mechanism that leads to its amplifica- 
Hepadnavirus Genome Replication and Persistence

tion (Tuttleman et al. 1986). Experiments with DHBV-infected ducks showed that certain envelope mutants accumulate cccDNA in much higher amounts than normal, which results in death of infected hepatocytes (Summers et al. 1990; Lenhoff and Summers 1994). In mammalian hepadnaviruses, the envelope proteins plus additional, so far unknown, factors are believed to play a role in the regulation of intracellular cccDNA amplification (Gao and $\mathrm{Hu}$ 2007; Guo et al. 2007; Lentz and Loeb 2011).

\section{THE RT}

\section{RT Structure and Functions}

RT has an approximate molecular weight (MW) of $90 \mathrm{kd}$ and contains four domains: $\mathrm{TP}$, a spacer, the polymerase/RT domain, and RNase H (Fig. 5) (Toh et al. 1983; Radziwill et al. 1990). The TP domain is unique to hepadnaviruses and does not share any apparent amino acid sequence similarities or known structural features with other viral or cellular proteins. TP carries the tyrosine residue that is essential for the priming of minus-strand DNA synthesis (Weber et al. 1994; Zoulim and Seeger 1994; Lanford et al. 1997; Jones et al. 2012) and, together with the polymerase domain, is required for binding to the $\varepsilon$ RNA (Wang et al. 1994; $\mathrm{Hu}$ and Anselmo 2000; Hu and Boyer 2006). The spacer region is believed to provide a flexible hinge between the TP and polymerase domains of the RT. The latter harbors a YMDD motif, which is conserved across all RTs, and is essential for both RNA- and DNA-dependent DNA synthesis, including protein priming (Chang et al. 1990; Radziwill et al. 1990; Xiong and Eickbush 1990; Lanford et al. 1997; Jones et al. 2012). The RNase $H$ domain at the carboxyl terminus of the RT contains a stretch of $\sim 100$ amino acids harboring a "D-E-D-D" catalytic motif known to coordinate metal ion binding (Nowotny et al. 2005). As mentioned earlier, the RNase $\mathrm{H}$ activity degrades pgRNA during minus-strand DNA synthesis and exerts a critical function in the formation of the primer for plus-strand DNA synthesis (Chang et al. 1990; Radziwill et al. 1990; Chen and Marion 1996; Tavis et al. 2013). Because the RT remains attached to the $5^{\prime}$ end of minus-strand DNA in infectious virus particles, it is possible that it plays a final role in the conversion of rcDNA into cccDNA, as alluded to above.

In addition to its enzymatic function, RT is also an essential mediator of $\varepsilon-\mathrm{RT}$ binding, protein priming, and RNA packaging into functional nucleocapsids. However, the structural requirements differ among the three reactions. For example, genetic analyses of the HBV RT

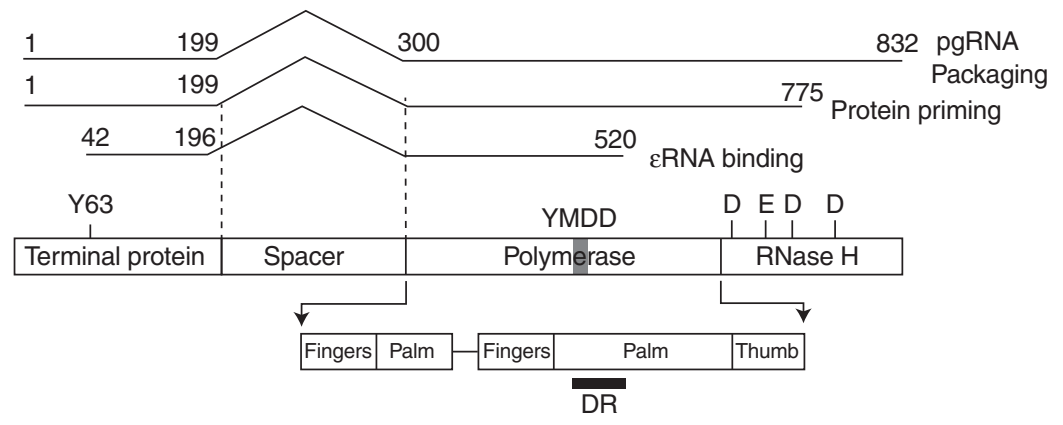

Figure 5. Physical and functional map of the hepatitis B virus (HBV) reverse transcriptase (RT). The figure shows the four domains of the RT (terminal protein, spacer, polymerase, and RNase H) described in the text. The predicted structural subdomains of the polymerase domain (fingers, palm, and thumb) are based on comparison with the crystal structure of human immunodeficiency virus (HIV) RT (Das et al. 2001). The location of mutations that confer drug resistance to nucleotide analogs currently used in antiviral therapy is indicated (drug resistance, DR). The regions of the RT required for RNA packaging, protein priming, and binding to $\varepsilon$ RNA are shown above the figure (see text for details). 
J. Hu and C. Seeger

revealed multiple residues in the TP and polymerase domains that are dispensable for binding to $\varepsilon$ RNA, but are critical for RNA packaging and protein priming (Shin et al. 2011a,b; Jones et al. 2014). In contrast to RNA packaging, the in vitro protein-priming reaction does not depend on a structurally intact RNase $\mathrm{H}$ domain (Fig. 5) (Bartenschlager et al. 1990; Hirsch et al. 1990; Chen et al. 1994; Jones et al. 2014). It is conceivable that the RNase domain is required for an interaction with the core protein during the assembly of core particles that are competent for genome replication. Finally, neither polymerase nor RNase $\mathrm{H}$ activities are required for RNA packaging (Bartenschlager et al. 1990; Hirsch et al. 1990; Jones et al. 2012; Cao et al. 2013; Jones and $\mathrm{Hu} 2013$ ).

\section{Requirements for $\varepsilon$ in pgRNA Packaging and Protein Priming}

As described earlier in this review, $\varepsilon$ RNA is critical for the activities of RT related to RNA packaging and protein priming (Junker-Niepmann et al. 1990; Hirsch et al. 1991; Bartenschlager and Schaller 1992; Wang et al. 1994; Jones et al. 2012). The $\varepsilon$ structure consists of two short stems, formed by two inverted repeats, which are connected by an apical loop and an internal bulge (Fig. 4). Although pgRNA packaging and protein priming require the apical loop of $\varepsilon$, binding of RT does not (Knaus and Nassal 1993; Pollack and Ganem 1993, 1994; Hu et al. 2004; Jones et al. 2012). Differences also exist in the structural requirements for RNA packaging versus protein priming. For example, most of the internal bulge appears to play, mainly, a structural role in RNA packaging, whereas the exact sequence is important for minus-strand DNA synthesis, as described before (Wang and Seeger 1993; Tavis et al. 1994; Nassal and Rieger 1996). Also, certain mutations in the upper stem do not affect RNA packaging, but abolish DNA synthesis, presumably caused by a defect in protein priming (Fallows and Goff 1995).

HBV RNA packaging, as well as protein priming, also depend on the presence and location of the $5^{\prime}$ cap structure, which has to be separated by $<70$ nucleotides from $\varepsilon$ (Fig. 4)
(Jeong et al. 2000; Jones et al. 2012). In all known hepadnavirus genomes, $\varepsilon$ is always located upstream of the AUG initiation codon for translation of the core protein. In fact, translation of the nucleotide sequence encoding $\varepsilon$ abolishes its activity in RNA packaging. Most likely, the passage of $80 \mathrm{~S}$ ribosomes disrupts some structural features of $\varepsilon$ required for packaging. For this reason, messenger RNA (mRNA) for the precore protein (e-antigen), which initiates $\sim 30$ nucleotides upstream of pgRNA, is not a substrate for packaging and reverse transcription (Fig. 2) (Nassal et al. 1990).

\section{Host Factors Required for DNA Replication}

Early biochemical studies of hepadnavirus RT were thwarted by difficulties in obtaining an active independently expressed protein ( $\mathrm{Hu}$ and Seeger 1996a). It was not until 1992 that enzymatically active RT of DHBV was expressed in a rabbit reticulocyte lysate (RRL) in vitro translation system (Wang and Seeger 1992). With this system, it could be shown that $\varepsilon$ is the template for the initiation of DNA synthesis and tyrosine residue 96 in the DHBV TP domain served as a primer for this reaction. These results established that packaging and priming of reverse transcription are linked events in the hepadnavirus life cycle and both are triggered by the RT $-\varepsilon$ interaction. The observation that the enzymatic activity of the RT expressed in RRL was much higher than in the wheat germ in vitro translation systems indicated that certain host factors must be required for RT activity. This prediction was confirmed by the demonstration that molecular chaperones, consisting of heat shock protein 90 (Hsp90) and its cofactor p23 (PTGES3), are required for the formation of a ribonucleoprotein (RNP) complex between $\varepsilon$ RNA and RT, in an energy-dependent manner (Fig. 4) (Hu and Seeger 1996b; Hu et al. 1997). Validation of the results originally obtained with the help of a cell-free RRL system included the demonstration that geldanamycin, a known inhibitor of Hsp90, inhibited packaging of pgRNA into capsids in tissue culture cells. Furthermore, p23, a cochaperone of Hsp90, is 
incorporated into pgRNA-containing, but not empty capsids in cells replicating $\mathrm{DHBV}(\mathrm{Hu}$ et al. 1997).

Reconstitution experiments with RT proteins purified from bacterial expression systems and host chaperone proteins helped to define the minimal cellular requirements for RNP formation and protein priming ( $\mathrm{Hu}$ and $\mathrm{An}$ selmo 2000; Hu et al. 2002, 2004). Together with Hsp90, several other chaperone proteins, including Hsp70, Hsp40, Hop, and p23, all known components of the Hsp90 complex, are necessary for the specific binding of the RT to $\varepsilon$ in vitro (Hu et al. 2002, 2004). Such a reconstituted RT $-\varepsilon$ complex of DHBV (but not $\mathrm{HBV}$; see below) is then competent for the protein-priming reaction ( $\mathrm{Hu}$ and Anselmo 2000; Hu et al. 2002).

The precise function of chaperones in protein priming is not completely understood because high-resolution structural information about RT is still lacking. It is possible that the chaperones stabilize an energetically unfavorable RT conformation and, in so doing, facilitate its binding to $\varepsilon$ (Fig. 4). There is evidence that DHBV RTwith deletion of the entire RNase $\mathrm{H}$ domain and the carboxy-terminal portion of the polymerase domain has $\varepsilon$-binding and protein-priming activities in the absence of cellular chaperones (Wang et al. 2003). Thus, the carboxy-terminal domain might hold the RT in a "closed," inactive conformation that, in the absence of chaperones, prevents the interaction with $\varepsilon$. Two conserved short-sequence motifs at the carboxy-terminal region of TP (T3) and at the amino-terminal section of RT (RT1), as well as residues outside these motifs, are critical for the binding of RT to $\varepsilon$ (Fig. 5) (Cao et al. 2005, 2013; Badtke et al. 2009; Jones et al. 2014). The $\mathrm{T} 3$ motif resides in a region of the TP that is transiently exposed at the surface of RT following its ATP-dependent activation by the host chaperones (Stahl et al. 2007).

Once RT binds to $\varepsilon$, a presumably stabilized structure promotes the enzymatic activity required for the protein-priming reaction (Tavis and Ganem 1996; Tavis et al. 1998). The $\varepsilon$ RNA also undergoes structural changes as a result of RT binding, which are thought to be required for protein priming and, possibly, pgRNA packaging (Beck and Nassal 1997, 1998).

Based on current knowledge, we can envision a process in which initiation of reverse transcription and assembly of replication competent core particles requires at least three distinct steps, each of which depend on different signals on the RT and pgRNA: (1) the interaction of TP and polymerase domains with the internal bulge and the surrounding stem sequences of $\varepsilon$, (2) the priming of reverse transcription, which requires a functional TP and enzymatically active polymerase domains, as well as the bulge of $\varepsilon$ and, (3) encapsidation of the RNP complex with core subunits, which depend on structurally intact RT and $\varepsilon$ RNA.

\section{Differences between HBV and DHBV RTs}

HBV and DHBV RTs are structurally very similar, having the same domain organization and the same mechanism of protein priming. Nevertheless, they show some distinct properties. For example, HBV RT expressed in RRL does not show enzymatic activity, which is a major reason why most investigations described above were performed with DHBV. HBV RTexpressed in insect cells using baculovirus vectors shows some in vitro protein-priming activity. In this system, tyrosine 63 , corresponding to tyrosine 96 in DHBV, acts as a primer for DNA synthesis. However, the reaction did not depend on the presence of $\varepsilon$ RNA (Lanford et al. 1995, 1997). Although HBV RT isolated from human tissue culture cells in a complex with host chaperones can bind to $\varepsilon$ in vitro (Jones et al. 2012), protein-priming activity can only be detected when RT is isolated from cells as an RNP with $\varepsilon$. Furthermore, as mentioned above, $\mathrm{HBV}$ protein priming requires a closely spaced $5^{\prime}$ cap on $\varepsilon$, which is not needed for DHBV priming. Unlike DHBV RT, the carboxy-terminal portion of the HBV RT, including most of the RNase $\mathrm{H}$ domain, although dispensable for $\varepsilon$ RNA binding, is essential for protein priming (Lanford et al. 1999; Jones et al. 2014). Under conditions that are permissive for priming with DHBV RT, HBV RT can bind $\varepsilon$ RNA, but does not display any protein-priming activity ( $\mathrm{Hu}$ et al. 2004; $\mathrm{Hu}$ 
J. Hu and C. Seeger

and Boyer 2006). These and other observations indicate that additional, as yet elusive, cellular factors might be required for the HBV proteinpriming reaction.

\section{RT and Genome Variability}

HBV has been classified into eight different genotypes based on differences in nucleotide sequence that exceed $4 \%$ in the surface gene and $8 \%$ across the complete genome (Kramvis and Kew 2005). Sequence heterogeneity among $\mathrm{HBV}$ isolates is expected because the RTs have no proofreading activities and, as a consequence, show error rates that are $\sim 10,000$ times higher than those of cellular DNA polymerases during replication of chromosomal DNA. However, in contrast to HIV or hepatitis $\mathrm{C}$ virus, a positive-strand RNA virus, sequence variations observed within the virus population in an infected patient or experimentally infected animals, are often difficult to detect (Hannoun et al. 2000). One reason for the difficulty lies in the complexity of the small HBV genome that limits survival capacity of mutants. All viral genes are overlapped either by a second reading frame or regulatory signals, such as enhancers, promoters, and signals for RNA packaging and DNA replication (Fig. 2). Nevertheless, selective pressure exerted by the host immune system or antiviral therapy can result in the accumulation of genetic variants within an infected host. For example, vaccination of children can result in the selection of vaccine escape variants with mutations in S that do not jeopardize the function of the overlapping pol gene (Locarnini and Yuen 2010). Similarly, antiviral therapy can result in the emergence of drug-resistant variants that show mutations in the polymerase domain of the RT at sites known to play a role in the binding of nucleotide triphosphates (Scaglione and Lok 2012). Interestingly, two nucleos(t)ide analog RT inhibitors show the highest barriers to drug resistance: entecavir, a carbocyclic G analog, and tenofovir disoproxil fumarate, an acyclical A analog, which can also block the protein-priming reaction competitively (Gish et al. 2012; Jones et al. 2013). In fact, resistance to tenofovir has, so far, not been reported. How- ever, from these results it is difficult to extrapolate the actual natural mutation rate of hepadnaviruses because the degree of virus replication varies among patients as a result of differences in the activity of the immune response against infected hepatocytes and other unknown parameters.

For DHBV, the frequency of spontaneous mutation and, hence, the mutation rate, could be estimated from the appearance of noncytopathic DHBV derived from a cytopathic DHBV strain during experimental infections of ducklings (Pult et al. 2001). Under the selected conditions, the mutation rate was estimated to $\sim 2 \times 10^{-5}$ per nucleotide per generation. The number of genomes produced each day during an infection is about a millionfold higher, indicating that every possible mutation is likely present at the time when selection for a particular variant occurs. The rate at which the variants accumulate depends not only on inherent replication fitness, but also on the fraction of uninfected hepatocytes required for establishment and amplification of the mutant genomes.

Besides misincorporation of nucleotides into minus- and plus-strand DNA during DNA replication, deamination of cytidine residues by members of the apolipoprotein B mRNA-editing catalytic polypeptides (APOBECs) can lead to the production of viral genomes with $G$ to $A$ hypermutations (Janahi and McGarvey 2013). Whether this mechanism contributes to the production of viable viral mutants or plays any role in viral pathogenesis remains to be determined.

\section{VIRAL PERSISTENCE}

The availability of nucleos(t)ide analog RT inhibitors markedly slows the progression of chronic hepatitis B by suppressing DNA replication. Although antiviral therapy prevents de novo production of viral DNA, it does not directly affect existing viral products. Assuming that the RT can be completely blocked, the concentration of the viral DNA products is expected to decline as a function of their respective halflives. For example, viral titers in the blood of $\mathrm{HBV}$-infected patients treated with different 
RT inhibitors, including lamivudine, adefovir dipivoxil, and tenofovir disoproxil fumarate, declined rapidly within hours after drug administration, leading to an estimation of the $t_{1 / 2}$ for virus particles in the range of 12-36 h (Nowak et al. 1996; Tsiang et al. 1999; Lewin et al. 2001, 2009). Similar results have been reported from a study with woodchucks infected with the woodchuck hepatitis virus (WHV) and treated with entecavir (Genovesi et al. 1998). Experiments aimed at determining the decay rates of intracellular viral DNA during antiviral therapy are more difficult to perform in vivo because of the limited number of liver biopsies that can be performed on a single animal. For example, a study with chronically infected woodchucks treated with the RT inhibitor 1-(2-fluoro-5-methyl$\beta, \mathrm{L}$-arabinofuranosyl)uracil (L-FMAU) revealed that replicative DNA declined within the first 2 wk of treatment and dropped to barely detectable levels by 6 wk (Zhu et al. 2001). Although these results are consistent with the model for DNA replication, which predicts that DNA-containing core particles bind to envelope proteins and enter the secretory pathway, they could also indicate that RT inhibition is incomplete, resulting in the observed persistence of core DNA in the liver for several weeks posttreatment.

In contrast to replicative intermediates, the amount of cccDNA in infected livers declined at a slow rate, with an estimated half-life ranging from 30 to $50 \mathrm{~d}$, similar to that of hepatocytes (Zhu et al. 2001). Notably, the ratio of virus positive hepatocytes to cccDNA levels increased during antiviral therapy, indicating that the copy number of cccDNA in infected hepatocytes declined. As noted earlier in this review, infected hepatocytes harbor multiple copies of cccDNA per nucleus and, in theory, cccDNA cannot be reamplified if viral replication is blocked with an RT inhibitor. However, if the block is incomplete, it is conceivable that some cccDNA may be replenished by the intracellular cccDNA amplification pathway during antiviral therapy. Despite these reservations, the results are consistent with the interpretation that cccDNA is stable in resting hepatocytes and declines as a consequence of dilution through cell division. The persistence of cccDNA is mani- fested by the rapid rebound of viremia known to occur following termination of antiviral therapy in experimental animals, as well as in human patients (Genovesi et al. 1998).

In summary, based on all the evidence derived from observations with patients and experimental animals treated with the RT inhibitors that are currently available, it can be concluded that free virions decay much more rapidly than intracellular replicative intermediates. As the decay rate of cccDNA is similar to the death rate of infected hepatocytes, it is likely that cccDNA is stable in infected cells. Hence, cell death and dilution of cccDNA during cell division of infected hepatocytes must be primarily responsible for the observed depletion of cccDNA during antiviral therapy. As will be discussed in the following paragraphs, the scenario in transient infections, which elicit a strong immune response against virus-infected hepatocytes, might be more complex.

In contrast to currently available antiviral therapies, a strong immune response against $\mathrm{HBV}$-infected hepatocytes can cure an infection. Key questions in this case concern the mechanisms responsible for the loss of cccDNA and prevention of new cccDNA synthesis by intracellular amplification or de novo infection of hepatocytes. Investigations into the mechanism of viral clearance rely on experimental inoculation either of chimpanzees, woodchucks, or ducks, and the collection of multiple serum and liver samples during the course of such infections. Although there is uniform agreement that cccDNA levels decline as a consequence of elimination of infected hepatocytes by cytotoxic $\mathrm{T}$ cells, there is still a debate about the extent of noncytolytic destruction of cccDNA during the clearance phase (Fourel et al. 1994a; Murray et al. 2005; Mason et al. 2009). For example, based on the analyses of blood and tissue samples from three HBV-infected chimpanzees and data-fitting analysis of the immune clearance phase, it has been proposed that cccDNA has a half-life of $\sim 3 \mathrm{~d}$ during the clearance phase, implying that noncytolytic mechanisms could contribute to the overall loss of cccDNA (Murray et al. 2005). Experiments with transiently infected woodchucks revealed that as much as 
a complete liver equivalent of cells could be replaced because of killing by cytotoxic T cells within a few weeks during the recovery phase (Kajino et al. 1994; Guo et al. 2000; Summers et al. 2003). Mathematical modeling of data derived from cccDNA analyses of liver tissues from infected woodchucks also indicated that cccDNA loss could not be explained solely by dilution during cell division (Mason et al. 2009). But solid evidence for a noncytolytic mechanism leading to cccDNA loss has, so far, not been forthcoming. An intriguing possibility would be that host antiviral factors produced during the clearance phase could degrade cccDNA. In fact, a recent report suggested that IFN- $\alpha$ or activation of the lymphotoxin$\beta$-receptor could induce the production of APOBECs, resulting in cytidine deamination and, subsequently, degradation of cccDNA (Lucifora et al. 2014). On the other hand, it is conceivable that the rates for cell killing and regeneration of hepatocytes during the early phase of the clearance process are higher than estimated from the analyses of biopsied liver tissues, and, as a consequence, cccDNA is lost at a much higher rate than predicted from mathematical modeling. In this case, clearance would be possible without a specific antiviral activity against cccDNA. Finally, it is conceivable that in a fraction of cccDNA genomes, transcription is silenced by epigenetic mechanisms. This would explain why cccDNA can be detected in liver samples for months to years after apparent resolution of an acute infection (Murray et al. 2005).

To overcome the limitations imposed by experiments with animal models, tissue culture systems have been used to investigate the fate of cccDNA in resting and dividing cells. For example, experiments with primary woodchuck hepatocyte cultures infected with WHV and treated with different RT inhibitors, including (-)- $\beta$-L- $2^{\prime}, 3^{\prime}$-dideoxy- $3^{\prime}$-thiacytidine (3TC, lamivudine) and L-FMAU, to block viral DNA synthesis revealed that cccDNA had half-lives exceeding $30 \mathrm{~d}$, indicating that it was stable in nondividing cells (Moraleda et al. 1997; Zhu et al. 2001). In contrast, experiments with established tissue culture cell lines that produce DHBV and lamivudine showed that cccDNA is lost during cell division (Guo et al. 2003). Because this loss could be accounted for by dilution, it appears that at least some cccDNA can survive mitosis, essentially, as predicted from experiments with woodchucks treated with RT inhibitors described previously.

\section{PERSPECTIVES}

Information gained from investigations during the past 30 years has provided us with many details concerning the hepadnavirus replication cycle. Besides the function of the viral core and RT proteins, the role of host proteins in RNA packaging and reverse transcription have been revealed. However, important gaps in knowledge still exist. For example, we still lack structural information about the unique TP region of the RT, a potential target for the development of non-nucleoside RT inhibitors, enzymes, and other proteins required for cccDNA synthesis are still elusive, the fate of cccDNA remains contentious, and questions concerning the role of cytokines or any other factors in cccDNA destabilization remain unanswered. A better understanding of the factors that are required for the maintenance of cccDNA is critical for the design of future antiviral therapies that can cure chronic hepatitis B.

\section{ACKNOWLEDGMENTS}

We thank Sid Balachandran, William Mason, and Ann Skalka for critical reading of the manuscript and helpful comments. J.H. and C.S. acknowledge support by the National Institutes of Health and the Commonwealth of Pennsylvania.

\section{REFERENCES}

Abraham TM, Loeb DD. 2006. Base pairing between the $5^{\prime}$ half of $\varepsilon$ and a cis-acting sequence, $\Phi$, makes a contribution to the synthesis of minus-strand DNA for human hepatitis B virus. J Virol 80: 4380-4387.

Abraham TM, Loeb DD. 2007. The topology of hepatitis B virus pregenomic RNA promotes its replication. J Virol 81: 11577-11584.

Badtke MP, Khan I, Cao F, Hu J, Tavis JE. 2009. An interdomain RNA binding site on the hepadnaviral polymer- 
ase that is essential for reverse transcription. Virology 390: $130-138$.

Bartenschlager R, Schaller H. 1992. Hepadnaviral assembly is initiated by polymerase binding to the encapsidation signal in the viral RNA genome. EMBO J 11:3413-3420.

Bartenschlager R, Junker-Niepmann M, Schaller H. 1990. The $\mathrm{P}$ gene product of hepatitis $\mathrm{B}$ virus is required as a structural component for genomic RNA encapsidation. J Virol 64: 5324-5332.

Basagoudanavar SH, Perlman DH, Hu J. 2007. Regulation of hepadnavirus reverse transcription by dynamic nucleocapsid phosphorylation. J Virol 81: 1641-1649.

Beck J, Nassal M. 1997. Sequence- and structure-specific determinants in the interaction between the RNA encapsidation signal and reverse transcriptase of avian hepatitis B viruses. J Virol 71: 4971-4980.

Beck J, Nassal M. 1998. Formation of a functional hepatitis $\mathrm{B}$ virus replication initiation complex involves a major structural alteration in the RNA template. Mol Cell Biol 18: 6265-6272.

Calvert J, Summers J. 1994. Two regions of an avian hepadnavirus RNA pregenome are required in cis for encapsidation. J Virol 68: 2084-2090.

Cao F, Badtke MP, Metzger LM, Yao E, Adeyemo B, Gong Y, Tavis JE. 2005. Identification of an essential molecular contact point on the duck hepatitis B virus reverse transcriptase. J Virol 79: 10164-10170.

Cao F, Jones SA, Li W, Cheng X, Hu Y, Hu J, Tavis JE. 2013. Sequences in the terminal protein and reverse transcriptase domains of the hepatitis B virus polymerase contribute to RNA binding and encapsidation. J Viral Hepat 21: $882-893$.

Chang LJ, Hirsch RC, Ganem D, Varmus HE. 1990. Effects of insertional and point mutations on the functions of the duck hepatitis B virus polymerase. J Virol 64: 55535558.

Chen Y, Marion PL. 1996. Amino acids essential for RNase H activity of hepadnaviruses are also required for efficient elongation of minus-strand viral DNA. J Virol 70: 61516156.

Chen Y, Robinson WS, Marion PL. 1994. Selected mutations of the duck hepatitis B virus $\mathrm{P}$ gene RNase $\mathrm{H}$ domain affect both RNA packaging and priming of minus-strand DNA synthesis. J Virol 68: 5232-5238.

Cortes Ledesma F, El Khamisy SF, Zuma MC, Osborn K, Caldecott KW. 2009. A human 5' -tyrosyl DNA phosphodiesterase that repairs topoisomerase-mediated DNA damage. Nature 461: 674-678.

Das K, Xiong X, Yang H, Westland CE, Gibbs CS, Sarafianos SG, Arnold E. 2001. Molecular modeling and biochemical characterization reveal the mechanism of hepatitis B virus polymerase resistance to lamivudine (3TC) and emtricitabine (FTC). J Virol 75: 4771-4779.

Fallows DA, Goff SP. 1995. Mutations in the epsilon sequences of human hepatitis B virus affect both RNA encapsidation and reverse transcription. J Virol 69: 3067-3073.

Fourel I, Cullen JM, Saputelli J, Aldrich CE, Schaffer P, Averett DR, Pugh J, Mason WS. 1994a. Evidence that hepatocyte turnover is required for rapid clearance of duck hepatitis B virus during antiviral therapy of chronically infected ducks. J Virol 68: 8321-8330.

Fourel I, Saputelli J, Schaffer P, Mason WS. 1994b. The carbocyclic analog of $2^{\prime}$-deoxyguanosine induces a prolonged inhibition of duck hepatitis B virus DNA synthesis in primary hepatocyte cultures and in the liver. J Virol 68: $1059-1065$.

Gao W, Hu J. 2007. Formation of hepatitis B virus covalently closed circular DNA: Removal of genome-linked protein. J Virol 81: 6164-6174.

Genovesi EV, Lamb L, Medina I, Taylor D, Seifer M, Innaimo S, Colonno RJ, Standring DN, Clark JM. 1998. Efficacy of the carbocyclic $2^{\prime}$-deoxyguanosine nucleoside BMS200475 in the woodchuck model of hepatitis B virus infection. Antimicrob Agents Chemother 42: 3209-3217.

Gerelsaikhan T, Tavis J, Bruss V. 1996. Hepatitis B virus nucleocapsid envelopment does not occur without genomic DNA synthesis. J Virol 70: 4269-4274.

Gerlich WH, Robinson WS. 1980. Hepatitis B virus contains protein attached to the $5^{\prime}$ terminus of its complete DNA strand. Cell 21: 801-809.

Gish R, Jia JD, Locarnini S, Zoulim F. 2012. Selection of chronic hepatitis B therapy with high barrier to resistance. Lancet Infect Dis 12: 341-353.

Gong SS, Jensen AD, Wang H, Rogler CE. 1995. Duck hepatitis $\mathrm{B}$ virus integrations in LMH chicken hepatoma cells: Identification and characterization of new episomally derived integrations. J Virol 69: 8102-8108.

Guo J-T, Zhou H, Liu C, Aldrich C, Saputelli J, Whitaker T, Barrasa MI, Mason WS, Seeger C. 2000. Apoptosis and regeneration of hepatocytes during recovery from transient hepadnavirus infection. J Virol 74: 1495-1505.

Guo JT, Pryce M, Wang X, Barrasa MI, Hu J, Seeger C. 2003. Conditional replication of duck hepatitis B virus in hepatoma cells. J Virol 77: 1885-1893.

Guo H, Jiang D, Zhou T, Cuconati A, Block TM, Guo JT. 2007. Characterization of the intracellular deproteinized relaxed circular DNA of hepatitis B virus: An intermediate of covalently closed circular DNA formation. J Virol 81: 12472-12484.

Hanawalt PC, Spivak G. 2008. Transcription-coupled DNA repair: Two decades of progress and surprises. Nat Rev Mol Cell Biol 9: 958-970.

Hannoun C, Horal P, Lindh M. 2000. Long-term mutation rates in the hepatitis B virus genome. J Gen Virol 81: 7583.

Hirsch RC, Lavine JE, Chang LJ, Varmus HE, Ganem D. 1990. Polymerase gene products of hepatitis B viruses are required for genomic RNA packaging as well as for reverse transcription. Nature 344: 552-555.

Hirsch RC, Loeb DD, Pollack JR, Ganem D. 1991. cis-Acting sequences required for encapsidation of duck hepatitis B virus pregenomic RNA. J Virol 65: 3309-3316.

Hu J, Anselmo D. 2000. In vitro reconstitution of a functional duck hepatitis B virus reverse transcriptase: Posttranslational activation by Hsp90. J Virol 74: 11447-11455.

Hu J, Boyer M. 2006. Hepatitis B virus reverse transcriptase and $\varepsilon$ RNA sequences required for specific interaction in vitro. J Virol 80: 2141-2150. 
J. Hu and C. Seeger

Hu J, Seeger C. 1996a. Expression and characterization of hepadnavirus reverse transcriptases. Methods Enzymol 275: $195-208$.

Hu J, Seeger C. 1996b. Hsp90 is required for the activity of a hepatitis B virus reverse transcriptase. Proc Natl Acad Sci 93: 1060-1064.

Hu J, Toft DO, Seeger C. 1997. Hepadnavirus assembly and reverse transcription require a multi-component chaperone complex which is incorporated into nucleocapsids. EMBO J 16: 59-68.

Hu J, Toft D, Anselmo D, Wang X. 2002. In vitro reconstitution of functional hepadnavirus reverse transcriptase with cellular chaperone proteins. J Virol 76: 269-279.

Hu J, Flores D, Toft D, Wang X, Nguyen D. 2004. Requirement of heat shock protein 90 for human hepatitis B virus reverse transcriptase function. J Virol 78: 13122-13131.

Janahi EM, McGarvey MJ. 2013. The inhibition of hepatitis B virus by APOBEC cytidine deaminases. J Viral Hepat 20: $821-828$.

Jeong JK, Yoon GS, Ryu WS. 2000. Evidence that the $5^{\prime}$-end cap structure is essential for encapsidation of hepatitis B virus pregenomic RNA. J Virol 74: 5502-5508.

Jones SA, Hu J. 2013. Protein-primed terminal transferase activity of hepatitis B virus polymerase. J Virol 87: 2563 2576.

Jones SA, Boregowda R, Spratt TE, Hu J. 2012. In vitro $\varepsilon$ RNA-dependent protein priming activity of human hepatitis B virus polymerase. $J$ Virol 86: $5134-5150$.

Jones SA, Murakami E, Delaney W, Furman P, Hu J. 2013. Non-competitive inhibition of hepatitis B virus reverse transcriptase protein priming and DNA synthesis by the nucleoside analog clevudine. Antimicrob Agents Chemother 57: 4181-4189.

Jones SA, Clark DN, Cao F, Tavis JE, Hu J. 2014. Comparative analysis of hepatitis B virus polymerase sequences required for viral RNA binding, RNA packaging, and protein priming. J Virol 88: 1564-1572.

Junker-Niepmann M, Bartenschlager R, Schaller H. 1990. A short cis-acting sequence is required for hepatitis $\mathrm{B}$ virus pregenome encapsidation and sufficient for packaging of foreign RNA. EMBO J 9: 3389-3396.

Kajino K, Jilbert AR, Saputelli J, Aldrich CE, Cullen J, Mason WS. 1994. Woodchuck hepatitis virus infections: Very rapid recovery after a prolonged viremia and infection of virtually every hepatocyte. J Virol 68: 5792-5803.

Kaplan PM, Greenman RL, Gerin JL, Purcell RH, Robinson WS. 1973. DNA polymerase associated with human hepatitis B antigen. J Virol 12: 995-1005.

Kaplan PM, Ford EC, Purcell RH, Gerin JL. 1976. Demonstration of subpopulations of Dane particles. J Virol 17: 885-893.

Knaus T, Nassal M. 1993. The encapsidation signal on the hepatitis B virus RNA pregenome forms a stem-loop structure that is critical for its function. Nucleic Acids Res 21: 3967-3975.

Kramvis A, Kew MC. 2005. Relationship of genotypes of hepatitis $\mathrm{B}$ virus to mutations, disease progression and response to antiviral therapy. J Viral Hepat 12: 456-464.

Lanford RE, Notvall L, Beames B. 1995. Nucleotide priming and reverse transcriptase activity of hepatitis $B$ virus polymerase expressed in insect cells. J Virol 69: 4431-4439.
Lanford RE, Notvall L, Lee H, Beames B. 1997. Transcomplementation of nucleotide priming and reverse transcription between independently expressed TP and RT domains of the hepatitis B virus reverse transcriptase. J Virol 71: 2996-3004.

Lanford RE, Kim YH, Lee H, Notvall L, Beames B. 1999. Mapping of the Hepatitis B virus reverse transcriptase TP and RT domains by transcomplementation for nucleotide priming and by protein-protein interaction. $J$ Virol 73: $1885-1893$.

Lenhoff RJ, Summers J. 1994. Construction of avian hepadnavirus variants with enhanced replication and cytopathicity in primary hepatocytes. J Virol 68: 5706-5713.

Lentz TB, Loeb DD. 2011. Roles of the envelope proteins in the amplification of covalently closed circular DNA and completion of synthesis of the plus-strand DNA in hepatitis B virus. J Virol 85: 11916-11927.

Lewellyn EB, Loeb DD. 2007. Base pairing between cis-acting sequences contributes to template switching during plus-strand DNA synthesis in human hepatitis B virus. $J$ Virol 81: 6207-6215.

Lewin SR, Ribeiro RM, Walters T, Lau GK, Bowden S, Locarnini S, Perelson AS. 2001. Analysis of hepatitis B viral load decline under potent therapy: Complex decay profiles observed. Hepatology 34: 1012-1020.

Lewin SR, Ribeiro RM, Avihingsanon A, Bowden S, Matthews G, Marks P, Locarnini SA, Ruxrungtham K, Perelson AS, Dore GJ. 2009. Viral dynamics of hepatitis B virus DNA in human immunodeficiency virus-1-hepatitis B virus coinfected individuals: Similar effectiveness of lamivudine, tenofovir, or combination therapy. Hepatology 49: 1113-1121.

Lien JM, Aldrich CE, Mason WS. 1986. Evidence that a capped oligoribonucleotide is the primer for duck hepatitis B virus plus-strand DNA synthesis. J Virol 57: 229236.

Lien JM, Petcu DJ, Aldrich CE, Mason WS. 1987. Initiation and termination of duck hepatitis B virus DNA synthesis during virus maturation. J Virol 61: 3832-3840.

Liu N, Tian R, Loeb DD. 2003. Base pairing among three cisacting sequences contributes to template switching during hepadnavirus reverse transcription. Proc Natl Acad Sci 100: 1984-1989.

Locarnini SA, Yuen L. 2010. Molecular genesis of drugresistant and vaccine-escape HBV mutants. Antivir Ther 15: 451-461.

Loeb DL, Hirsch RC, Ganem D. 1991. Sequence-independent RNA cleavages generate the primers for plus strand DNA synthesis in hepatitis B viruses: Implications for other reverse transcribing elements. EMBO J 10: $3533-$ 3540 .

Lucifora J, Xia Y, Reisinger F, Zhang K, Stadler D, Cheng X, Sprinzl MF, Koppensteiner H, Makowska Z, Volz T, et al. 2014. Specific and nonhepatotoxic degradation of nuclear hepatitis B virus cccDNA. Science 343: 1221-1228.

Maguire ML, Loeb DD. 2010. cis-Acting sequences that contribute to synthesis of minus-strand DNA are not conserved between hepadnaviruses. J Virol 84: 12824-12831.

Mason WS, Xu C, Low HC, Saputelli J, Aldrich CE, Scougall C, Grosse A, Colonno R, Litwin S, Jilbert AR. 2009. The amount of hepatocyte turnover that occurred during resolution of transient hepadnavirus infections was lower 
when virus replication was inhibited with entecavir. $J$ Virol 83: 1778-1789.

Miller RH, Robinson WS. 1984. Hepatitis B virus DNA forms in nuclear and cytoplasmic fractions of infected human liver. Virology 137: 390-399.

Molnar-Kimber KL, Summers J, Taylor JM, Mason WS 1983. Protein covalently bound to minus-strand DNA intermediates of duck hepatitis B virus. J Virol 45: $165-172$.

Moraleda G, Saputelli J, Aldrich CE, Averett D, Condreay L, Mason WS. 1997. Lack of effect of antiviral therapy in nondividing hepatocyte cultures on the closed circular DNA of woodchuck hepatitis virus. J Virol 71: 9392 9399.

Mueller-Hill K, Loeb DD. 2002. cis-Acting sequences 5E, M, and $3 \mathrm{E}$ interact to contribute to primer translocation and circularization during reverse transcription of avian hepadnavirus DNA. J Virol 76: 4260-4266.

Murray JM, Wieland SF, Purcell RH, Chisari FV. 2005. Dynamics of hepatitis B virus clearance in chimpanzees. Proc Natl Acad Sci 102: 17780-17785.

Nassal M, Rieger A. 1996. A bulged region of the hepatitis B virus RNA encapsidation signal contains the replication origin for discontinuous first-strand DNA synthesis. $J$ Virol 70: 2764-2773.

Nassal M, Junker-Niepmann M, Schaller H. 1990. Translational inactivation of RNA function: Discrimination against a subset of genomic transcripts during HBV nucleocapsid assembly. Cell 63: 1357-1363.

Ning X, Nguyen D, Mentzer L, Adams C, Lee H, Ashley R, Hafenstein S, Hu J. 2011. Secretion of genome-free hepatitis B virus-Single strand blocking model for virion morphogenesis of para-retrovirus. PLoS Pathog 7: e1002255.

Nowak MA, Bonhoeffer S, Hill AM, Boehme R, Thomas HC, McDade H. 1996. Viral dynamics in hepatitis B virus infection. Proc Natl Acad Sci 93: 4398-4402.

Nowotny M, Gaidamakov SA, Crouch RJ, Yang W. 2005. Crystal structures of RNase $\mathrm{H}$ bound to an RNA/DNA hybrid: Substrate specificity and metal-dependent catalysis. Cell 121: 1005-1016.

Perlman D, Hu J. 2003. Duck hepatitis B virus virion secretion requires a double-stranded DNA genome. J Virol 77: 2287-2294.

Pollack JR, Ganem D. 1993. An RNA stem-loop structure directs hepatitis B virus genomic RNA encapsidation. J Virol 67: 3254-3263.

Pollack JR, Ganem D. 1994. Site-specific RNA binding by a hepatitis B virus reverse transcriptase initiates two distinct reactions: RNA packaging and DNA synthesis. $J$ Virol 68: 5579-5587.

Pult I, Abbott N, Zhang YY, Summers JW. 2001. Frequency of spontaneous mutations in an avian hepadnavirus infection. J Virol 75: 9623-9632.

Radziwill G, Tucker W, Schaller H. 1990. Mutational analysis of the hepatitis B virus P gene product: Domain structure and RNase H activity. J Virol 64: 613-620.

Roth MJ, Brown DR, Hurwitz J. 1984. Analysis of bacteriophage phi X174 gene A protein-mediated termination and reinitiation of phi X DNA synthesis. II. Structural characterization of the covalent phi X A protein-DNA complex. J Biol Chem 259: 10556-10568.

Rumin S, Gripon P, Le Seyec J, Corral-Debrinski M, Guguen-Guillouzo C. 1996. Long-term productive episomal hepatitis B virus replication in primary cultures of adult human hepatocytes infected in vitro. $J$ Viral Hepat 3: 227-238.

Scaglione SJ, Lok AS. 2012. Effectiveness of hepatitis B treatment in clinical practice. Gastroenterology 142: 1360 1368.e1.

Seeger C, Ganem D, Varmus HE. 1986. Biochemical and genetic evidence for the hepatitis $\mathrm{B}$ virus replication strategy. Science 232: 477-484.

Seeger C, Leber EH, Wiens LK, Hu J. 1996. Mutagenesis of a hepatitis B virus reverse transcriptase yields temperaturesensitive virus. Virology 222: 430-439.

Seeger C, Zoulim F, Mason WS. 2013. Hepadnaviruses. Lippincott, Williams and Wilkins, Philadelphia.

Shin YC, Ko C, Ryu WS. 2011a. Hydrophobic residues of terminal protein domain of hepatitis B virus polymerase contribute to distinct steps in viral genome replication. FEBS Lett 585: 3964-3968.

Shin YC, Park S, Ryu WS. 2011b. A conserved arginine residue in the terminal protein domain of hepatitis $B$ virus polymerase is critical for RNA pre-genome encapsidation. J Gen Virol 92: 1809-1816.

Sohn JA, Litwin S, Seeger C. 2009. Mechanism for CCC DNA synthesis in hepadnaviruses. PLoS ONE 4: e8093.

Stahl M, Beck J, Nassal M. 2007. Chaperones activate hepadnavirus reverse transcriptase by transiently exposing a $\mathrm{C}$-proximal region in the terminal protein domain that contributes to $\varepsilon$ RNA binding. J Virol 81: 13354-13364.

Staprans S, Loeb DD, Ganem D. 1991. Mutations affecting hepadnavirus plus-strand DNA synthesis dissociate primer cleavage from translocation and reveal the origin of linear viral DNA. J Virol 65: 1255-1262.

Summers J, Mason WS. 1982. Replication of the genome of a hepatitis B-like virus by reverse transcription of an RNA intermediate. Cell 29: 403-415.

Summers J, O'Connell A, Millman I. 1975. Genome of hepatitis $B$ virus: Restriction enzyme cleavage and structure of DNA extracted from Dane particles. Proc Natl Acad Sci 72: 4597-4601.

Summers J, Smith PM, Horwich AL. 1990. Hepadnavirus envelope proteins regulate covalently closed circular DNA amplification. J Virol 64: 2819-2824.

Summers J, Smith PM, Huang MJ, Yu MS. 1991. Morphogenetic and regulatory effects of mutations in the envelope proteins of an avian hepadnavirus. J Virol 65: 13101317.

Summers J, Jilbert AR, Yang W, Aldrich CE, Saputelli J, Litwin S, Toll E, Mason WS. 2003. Hepatocyte turnover during resolution of a transient hepadnaviral infection. Proc Natl Acad Sci 100: 11652-11659.

Tagawa M, Omata M, Okuda K. 1986. Appearance of viral RNA transcripts in the early stage of duck hepatitis B virus infection. Virology 152: 477-482.

Tavis JE, Ganem D. 1996. Evidence for activation of the hepatitis B virus polymerase by binding of its RNA template. J Virol 70: $5741-5750$ 
J. Hu and C. Seeger

Tavis JE, Perri S, Ganem D. 1994. Hepadnavirus reverse transcription initiates within the stem-loop of the RNA packaging signal and employs a novel strand transfer. J Virol 68: 3536-3543.

Tavis JE, Massey B, Gong Y. 1998. The duck hepatitis B virus polymerase is activated by its RNA packaging signal, $\varepsilon$. $J$ Virol 72: 5789-5796.

Tavis JE, Cheng X, Hu Y, Totten M, Cao F, Michailidis E, Aurora R, Meyers MJ, Jacobsen EJ, Parniak MA, et al. 2013. The hepatitis $B$ virus ribonuclease $H$ is sensitive to inhibitors of the human immunodeficiency virus ribonuclease $\mathrm{H}$ and integrase enzymes. PLoS Pathog 9: e1003125.

Toh H, Hayashida H, Miyata T. 1983. Sequence homology between retroviral reverse transcriptase and putative polymerases of hepatitis B virus and cauliflower mosaic virus. Nature 305: 827-829.

Tsiang M, Rooney JF, Toole JJ, Gibbs CS. 1999. Biphasic clearance kinetics of hepatitis B virus from patients during adefovir dipivoxil therapy. Hepatology 29: $1863-$ 1869.

Tuttleman JS, Pourcel C, Summers J. 1986. Formation of the pool of covalently closed circular viral DNA in hepadnavirus-infected cells. Cell 47: 451-460.

Wang GH, Seeger C. 1992. The reverse transcriptase of hepatitis B virus acts as a protein primer for viral DNA synthesis. Cell 71: 663-670.

Wang GH, Seeger C. 1993. Novel mechanism for reverse transcription in hepatitis B viruses. J Virol 67: 65076512.

Wang GH, Zoulim F, Leber EH, Kitson J, Seeger C. 1994. Role of RNA in enzymatic activity of the reverse transcriptase of hepatitis B viruses. J Virol 68: 84378442.

Wang X, Qian X, Guo HC, Hu J. 2003. Heat shock protein 90-independent activation of truncated hepadnavirus reverse transcriptase. J Virol 77: 4471-4480.
Weber M, Bronsema V, Bartos H, Bosserhoff A, Bartenschlager R, Schaller H. 1994. Hepadnavirus P protein utilizes a tyrosine residue in the TP domain to prime reverse transcription. J Virol 68: 2994-2999.

Wei Y, Tavis JE, Ganem D. 1996. Relationship between viral DNA synthesis and virion envelopment in hepatitis B viruses. J Virol 70: 6455-6458.

Wu TT, Coates L, Aldrich CE, Summers J, Mason WS. 1990. In hepatocytes infected with duck hepatitis B virus, the template for viral RNA synthesis is amplified by an intracellular pathway. Virology 175: 255-261.

Xiong Y, Eickbush TH. 1990. Origin and evolution of retroelements based upon their reverse transcriptase sequences. EMBO J 9: 3353-3362.

Yang W, Summers J. 1995. Illegitimate replication of linear hepadnavirus DNA through nonhomologous recombination. J Virol 69: 4029-4036.

Yang W, Summers J. 1998. Infection of ducklings with virus particles containing linear double-stranded duck hepatitis B virus DNA: Illegitimate replication and reversion. $J$ Virol 72: 8710-8717.

Yang W, Mason WS, Summers J. 1996. Covalently closed circular viral DNA formed from two types of linear DNA in woodchuck hepatitis virus-infected liver. J Virol 70: $4567-4575$.

Yu M, Summers J. 1991. A domain of the hepadnavirus capsid protein is specifically required for DNA maturation and virus assembly. J Virol 65: 2511-2517.

Zhu Y, Yamamoto T, Cullen J, Saputelli J, Aldrich CE, Miller DS, Litwin S, Furman PA, Jilbert AR, Mason WS. 2001. Kinetics of hepadnavirus loss from the liver during inhibition of viral DNA synthesis. J Virol 75: 311-322.

Zoulim F, Seeger C. 1994. Reverse transcription in hepatitis $B$ viruses is primed by a tyrosine residue of the polymerase. J Virol 68: 6-13. 


\section{$\&_{\mathrm{CSH}}^{\infty} \&$ Cold Spring Harbor

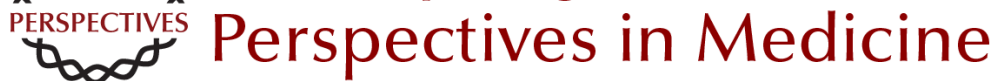

\section{Hepadnavirus Genome Replication and Persistence}

Jianming $\mathrm{Hu}$ and Christoph Seeger

Cold Spring Harb Perspect Med 2015; doi: 10.1101/cshperspect.a021386

Subject Collection The Hepatitis B and Delta Viruses

Hepatitis B Virus X and Regulation of Viral Gene Expression

Betty L. Slagle and Michael J. Bouchard

The Woodchuck, a Nonprimate Model for Immunopathogenesis and Therapeutic Immunomodulation in Chronic Hepatitis B Virus Infection

Michael Roggendorf, Anna D. Kosinska, Jia Liu, et al.

Mouse Models of Hepatitis B Virus Pathogenesis Matteo lannacone and Luca G. Guidotti

Therapy of Delta Hepatitis

Cihan Yurdaydin and Ramazan Idilman

Immune Response in Hepatitis B Virus Infection Anthony Tan, Sarene Koh and Antonio Bertoletti

Hepatitis D Virus: Introduction and Epidemiology Mario Rizzetto

Management of Chronic Hepatitis B in Patients from Special Populations

Ching-Lung Lai and Man-Fung Yuen

Hepatitis B Virus Genotypes and Variants

Chih-Lin Lin and Jia-Horng Kao
Origins and Evolution of Hepatitis B Virus and Hepatitis D Virus

Margaret Littlejohn, Stephen Locarnini and Lilly Yuen

Assembly and Release of Hepatitis B Virus Lisa Selzer and Adam Zlotnick

Hepatitis D Virus Replication John M. Taylor

Treatment of Liver Cancer

Chun-Yu Liu, Kuen-Feng Chen and Pei-Jer Chen

Hepatitis B Virus and Hepatitis D Virus Entry, Species Specificity, and Tissue Tropism

Koichi Watashi and Takaji Wakita

Hepadnavirus Genome Replication and

Persistence Jianming Hu and Christoph Seeger

The Chimpanzee Model for Hepatitis B Virus Infection

Stefan F. Wieland

Hepatitis B Virus Epidemiology

Jennifer H. MacLachlan and Benjamin C. Cowie

For additional articles in this collection, see http://perspectivesinmedicine.cshlp.org/cgi/collection/ 